\title{
DETERMINATION OF SNOW FACIES AND ICE VELOCITY USING SYNTHETIC APERTURE RADAR IMAGERY
}

\author{
(Abstract) \\ by \\ R.A. Bindschadler \\ (Code 671, Laboratory for Oceans, NASA/Goddard Space Flight Center, \\ Greenbelt, MD 20771, U.S.A.) \\ and \\ P.L. Vornberger
}

(STX, 4400 Forbes Boulevard, Lanham, MD 20706, U.S.A.)

The properties of synthetic aperture radar (SAR) imagery are appropriate for its use to map snow facies. These facies, defined by Benson (1962), are subdivisions of the accumulation area of an ice sheet or polar glacier and represent the interaction of the ice mass with the climate through the processes of snow accumulation and melting. Changes in these climatic parameters are expected to cause changes in the extent and character of these facies. The ability of SAR to discriminate these facies is due to the significant amount of sub-surface volume scattering in the measured radar backscatter signal and the strong absorption of radar energy by liquid water. The amount of volume scattering is dependent on the size and distribution of scatterers in the medium. This dependence varies over the size range of snow grains to ice lenses. Specific examples of the ability to detect different scatterer populations in ice sheets with SAR are shown. Other examples are given to demonstrate the reduction of backscatter signal when liquid water is present.
Another important application of SAR data is the determination of surface velocity. Coregistration of a SAR and a TM image spanning an eight-year period was completed for an area in south-western Greenland. The composite image shows that, while the network of surface streams is nearly unchanged, their distance from lakes upstream increased over the eight-year interval between images. Because the lakes are likely fixed in space, a result of surface depressions whose positions are determined by the stationary bedrock topography, the displacement of the stream network was used to calculate a surface velocity of $40 \pm 10 \mathrm{~m}$ per year near the equilibrium line.

\section{REFERENCE}

Benson, C.S. 1962. Stratigraphic studies in the snow and firn of the Greenland ice sheet. U.S. Army Snow Ice and Permafrost Research Establishment. Research Report 70.

\section{DIVERSE LIMITING CIRCULATIONS IN A SIMPLE OCEAN BOX MODEL \\ (Abstract)}

by

Edward Birchfield and Matthew Wyant

(Department of Geological Sciences, Northwestern University, Evanston, IL 60201, U.S.A.)

water produced in high latitudes, resembling the modern ocean; for a strong hydrological cycle, a warm saline deep ocean is found with deep water produced in lower latitudes, similar to proposed models of a Cretaceous ocean. More complex solutions exist for an intermediate range of parameters. These include co-existence of both of the above limiting circulations as stable steady states and an oscillatory solution about the cold deep-ocean limit case. In general for this model, the cold deep-ocean case appears less stable than the warm saline deep-ocean case. 